\title{
A New On-Line/Off-Line Adaptive Antenna Array Beamformer for Tracking the Mobile Targets
}

\author{
Shahriar Shirvani-Moghaddam ${ }^{1}$, Mahyar Shirvani-Moghaddam ${ }^{2}$ \\ ${ }^{1}$ Digital Communications Signal Processing Research Lab., Faculty of Electrical and Computer Engineering, \\ Shahid Rajaee Teacher Training University, Tehran, Iran \\ ${ }^{2}$ Telecommunication Laboratory, School of Electrical and Information, University of Sydney, Sydney, Australia \\ E-mail:sh_shirvani@srttu.edu,m.shirvanimoghaddam@sydney.edu.au \\ Received February 26, 2011; revised April 7, 2011; accepted April 23, 2011
}

\begin{abstract}
An adaptive antenna array system adjusts the main lobe of radiation pattern in the direction of desired signal and points the nulls in the direction of undesired signals or interferers. The essential goal of beamforming is to reduce the complexity of weighting process and to decrease the time needed for adjusting the antenna radiation pattern. In this article a new adaptive weighting algorithm is proposed for both least mean squares (LMS) and constant modulus (CM) algorithms. It is appropriate and applicable for antenna array systems with moving targets and also mobile applications as well as sensor networks. By predicting the relative velocity of source, the next location of the source will be estimated and the array weights will be determined using LMS or CM algorithm before arriving to the new point. For the next time associated to the new sampling point, evaluated weights will be used. Furthermore, by updating these weights between two consecutive times the effects of error propagation will be eliminated. Therefore, in addition to reduction in computational complexity at the time of weight allocation, relatively accurate weight allocation can be obtained. Simulation results of this investigation show that the angular error related to both LMS-based and CM-based algorithms is less than the conventional LMS and CM algorithms at different signal to noise ratios (SNRs). On the other hand, due to considering off-line process, online computational complexity of new algorithms is slightly low with respect to previous ones.
\end{abstract}

Keywords: Beamforming, Adaptive Antenna Array, LMS, CM, Training-Based, Blind

\section{Introduction}

Growth in wireless technologies and users' demands, lead us to extend these systems in rural and urban areas, indoor and outdoor environments, short-range as well as long-range applications and then optimizing them for long term scheduling. During these two decades, appropriate beamforming and concentrating radiated power in specific directions using antenna (sensor) arrays are been so attractive [1]. In digital beamforming, instead of hardware changes, major part of processing is done by digital processors in intermediate frequency (IF) or baseband. This type of processing increases the flexibility and reduces the size of the system. By appropriate beamforming at the transmitter, receiver or both, we can reach to higher receiving power, better signal to noise plus interference ratio (SNIR), and lower bit error rate (BER).
There are two major techniques for forming the radiation pattern of antenna arrays, fixed beam and adaptive processing [2-4]. As shown in fixed-beam methods, statistically optimum weight vectors for beamforming can be calculated by the Wiener solution. However, knowledge of the asymptotic second-order statistics of the signal and the interference-plus-noise should be assumed. These statistics are usually not known but with the assumption of ergodicity, where the time average equals the ensemble average, they can be estimated from the available data. For time-varying signal environments, such as wireless cellular communication systems, statistics change with time as the source and interferers move around the cell. For the time-varying signal propagation environment, a recursive update of the weight vector is needed to track a moving mobile or terminal so that the spatial beam filtering will adaptively steer to the target 
mobile's time-varying direction of arrival (DOA), thus resulting in optimal transmission/reception of the desired signal. To solve the problem of time-varying statistics, weight vectors are typically determined by adaptive algorithms which adapt to the changing environment $[5,6]$. In adaptive beamforming, according to the system condition, antenna array and its pattern will be adjusted dynamically. Thus, in this system, there is a processing unit. Types of antennas, their configurations and forwarding information to the processor depend on the application.

There are two different methods, based on training sequences and blind estimation, for adaptive weighting of antenna array elements. In training based methods one reference signal is required and they do not need to know the location of signal source and hence they will be converged faster than blind algorithms. In contrast, in blind methods, the only thing that is required is the DOA of the main signal (source) and other information should be obtained from received signal $[7,8]$.

In recent years different research works have been done in the field of smart antenna and digital beamforming. Reviewing these works shows that the effect of the direction and velocity of the source has not been considered seriously and if it has been used, calculations and computational complexity in the weighting step have been increased. For example, in [9], authors propose a normalized least mean square (NLMS) adaptive algorithm that incorporates a direction of arrival detection criterion. Simulation results of this investigation show that the number of NLMS adapted parameters can be reduced by this method. This method provides significantly improved convergence and tracking capabilities.

Authors in [10] shows that by using adaptive beamforming technique based on maximum likelihood estimation (MLE) robustness of algorithms against the effects of incorrect direction of arrival (DOA) estimation can be increased. In [11], the performance of an autoregressive (AR), super resolution beamforming technique is analyzed and compared with other high resolution methods. The AR parameters in [11] are determined adaptively using the least mean square (LMS) algorithm.

Practical design of a smart antenna system based on DOA estimation and adaptive beamforming is the subject of [12]. In [12] DOA estimation is based on multiple signal classification (MUSIC) algorithm and adaptive beamforming is achieved using the LMS algorithm.

In [13] the performance of LMS and recursive least square (RLS) algorithms for smart antennas in a wideband code division multiple access (W-CDMA) mobile communication environment is discussed. Furthermore, beamforming algorithms based on NLMS and matrix inverse (MI) are processed in [14]. In [15] a matrix inversion normalized least mean square (MI-NLMS) adap- tive beamforming algorithm is described with tracking. Simulation results of this method show that BER improvement is proportional to the number of antenna elements employed in the antenna array. Performance of constant modulus (CM), LMS, and RLS algorithms are discussed in [16] and a systematic comparison between them is obtained. The linearly constrained constant modulus algorithm (LCCMA) is the subject of [17]. In [18, 19], the performance of blind adaptive beamforming algorithms for smart antennas in realistic environments with a constrained constant modulus (CCM) design criterion is described and used for deriving a RLS type optimization algorithm. Ref. [20] shows a systematic comparison of the performance of different modified blind adaptive beamforming algorithms based on CCM. Two of them use adaptive step size mechanisms, in the stochastic Gradient (SG) algorithm for adjusting the step size and other one uses RLS type optimization algorithm which is replaced by inverse of correlation matrix instead of step size.

In the present article, by using LMS and CM algorithms, a new simple method for predicting the direction and velocity of source has been proposed. In comparison with other algorithms and also previous research works, it adds no additional calculations and complexity in the time of radiation pattern shaping (denoted as online process) and the main calculations are done by a separate processor in the time intervals between two consecutive times (denoted as offline process). Another advantage of the proposed algorithm is the simplicity of the estimation of the DOA related to the conventional DOA algorithms.

The rest of this paper is organized as follow. In Section 2, some important basic notes about adaptive antenna array systems are illustrated and two well-known algorithms, LMS and CM, are discussed in detail. Section 3 introduces the new proposed algorithm which is based on prediction the next location of source and doing the required processes in two steps, on-line as well as off-line. In addition to simulation assumptions and related flowchart, the simulation results of conventional LMS and CM as well as proposed ones are described in Section 4. Finally, Section 5 concludes this article.

\section{Adaptive Antenna Array Systems}

Antenna array is a set of antennas connected to a digital signal processor which are used for transmitting and receiving electromagnetic waves in coherent manner. Each antenna is an element of array. The transmitting beam in transmitter and/or receiving pattern in receiver can be formed using a processor in based-band by combining the array element signals. This process is called digital beamforming. Two most important parameters for deter- 
mining the radiation pattern of antenna arrays are the weighting method in reception and the type of feeding the elements in transmission. Hence some adaptive methods have been proposed for producing appropriate radiation patterns.

Assume a k-element antenna array as depicted in Figure 1. In order to obtain an appropriate radiation pattern, signals of array elements should be multiplied in the complex weights and weighted signals should be combined linearly. This process is called weighting. After receiving the signal at element $A n t_{1}$, it is multiplied with weight $w_{1}$ which modify the amplitude and phase of input signal. Then, according to (1), a linear system combines these weighted signals to create output signal.

$$
y(n)=w_{1} \cdot x_{1}(n)+w_{2} \cdot x_{2}(n)+\cdots+w_{k} \cdot x_{k}(n)
$$

The main feature of antenna array is the ability to separate signals in space. Signal separation in space means adjusting the main lobe of antenna in the direction of desired signal (main signal) and nulls in the direction of undesired signals (interferers). Therefore, input and output signals should be compared. In this investigation, LMS and CM algorithms are considered to find the best weights of antenna array system.

\subsection{LMS Algorithm}

LMS algorithm uses minimum mean square error (MMSE) criterion for weight estimation. In MMSE, if $E\left\{\varepsilon^{2}(t)\right\}$ is considered as a function of $w$, this function has only one minimum (bowl-shape surface). The location of this minimum can be determined by solving the Wyner optimization equation. Figure 2 shows the bowl- shape surface for two-weight function.

It is also known that the gradient of this surface, i.e., $\nabla_{w} E\left\{\varepsilon^{2}(t)\right\}$, is a vector directed to the maximum point of surface with minimum distance. Therefore, it will be moved to the minimum point with minimum distance, if it is altered the weights according to the negative slope of this vector. This method is called steepest-descent. This is the best choice to move toward the minimum point. This fact can be proven by stronger mathematical methods.

One of the interesting features of this method is that when we are close to the minimum point, the slope of gradient vector is high, and by increasing the distance to this point the slope of this vector is decreased. This feature avoids weight oscillation and instability due to variations of time variant channels.

According to the above mentioned descriptions, it can be summarized as follow:

$$
\frac{\mathrm{d} w}{\mathrm{~d} t}=-\mu \cdot \nabla_{w} E\left\{\varepsilon^{2}(t)\right\}
$$

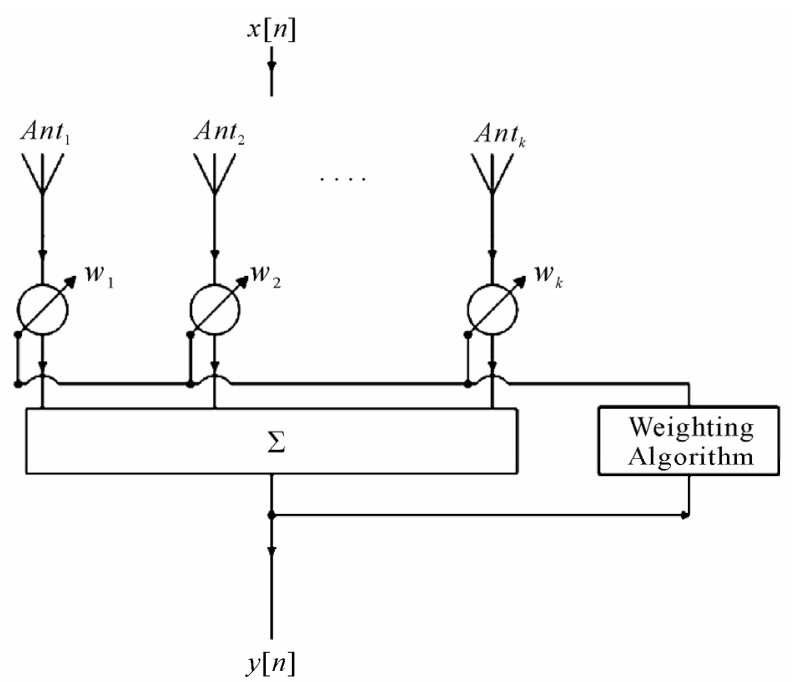

Figure 1. Adaptive antenna array structure.

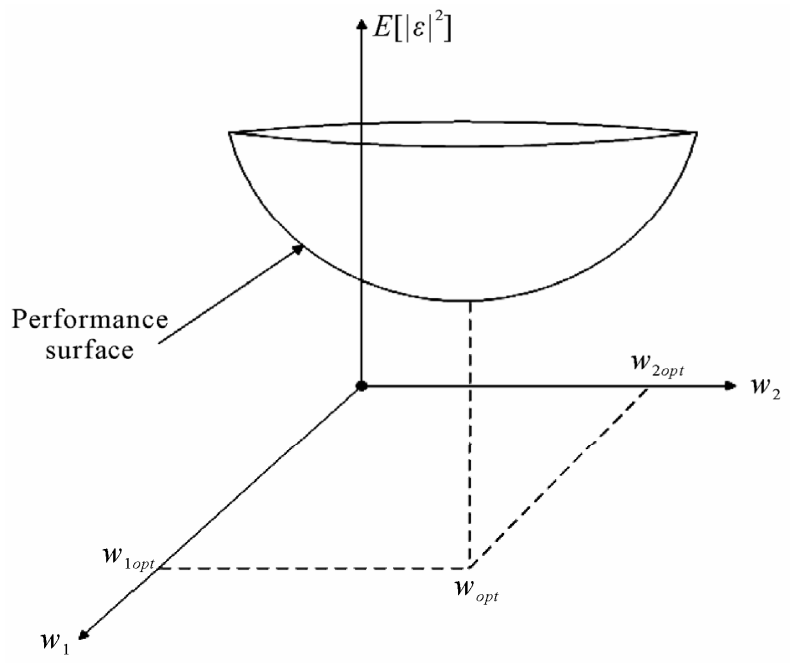

Figure 2. The surface of a two-weight array system.

$$
\begin{gathered}
E\left\{\varepsilon^{2}(t)\right\}=E\left\{d^{2}(t)\right\}-2 w^{H} \cdot r+w^{H} \cdot R \cdot w \\
\frac{\mathrm{d} w}{\mathrm{~d} t}=-2 \mu \cdot(-r+R \cdot w) \\
r=E\left\{d^{*}(t) \cdot x(t)\right\}, R=E\left\{x(t) \cdot x^{H}(t)\right\} \\
\frac{\mathrm{d} w}{\mathrm{~d} t}=2 \mu \cdot E\left\{x(t) \cdot \varepsilon^{*}(t)\right\}, \varepsilon(t)=y(t)-d(t)
\end{gathered}
$$

where $\mu$ is a coefficient that determines the convergence speed of the algorithm, $d(t)$ represents reference signal, and $H$ indicates Hermitian conjugate.

In (6) we use the real-time value instead of average value. This simplification is shown in (7).

$$
\frac{\mathrm{d} w}{\mathrm{~d} t}=2 \mu \cdot x(t) \cdot \varepsilon^{*}(t)
$$


For discrete-time case, derivations can be substituted with subtraction as follow:

$$
w(n+1)=w(n)+\mu \cdot x(n) \cdot \varepsilon^{*}(n)
$$

Equation (8) shows that using real-time approximation instead of expected value can introduce an iterative algorithm with simpler implementation. However, some notes should be considered about convergence speed. The surface $E\left\{\varepsilon^{2}(t)\right\}$ is the bowl-shaped which its shape is determined with eigen values of output correlation matrix of array. The crater of bowl will be too open and the slope of its walls will be small, if this eigen values are far together. Therefore, the convergence speed will be decreased.

\subsection{Algorithm}

In some techniques that modulate information in phase (such as M-ary phase shift keying (MPSK)) or frequency (such as M-ary frequency shift keying (MFSK)), the envelope of signal remains constant. The CM algorithm is first proposed by Godard and it uses this constant envelope feature. By calculating this envelope, adaptive beamforming algorithm can be managed.

CM algorithm uses a cost function, named as diffraction function of order $\mathrm{p}$, and after minimization, the optimum weights can be obtained. The Godard's cost function is shown in (9).

$$
J(n)=E\left\{\left(|y(n)|^{p}-R_{p}\right)^{2 q}\right\}
$$

where $p, q$ are equal to 1 or 2 . Godard showed that if $R_{p}$ is defined as in (10), the slope of the cost function will be zero.

$$
R_{p}=\frac{E\left\{|s(n)|^{2 p}\right\}}{E\left\{|s(n)|^{p}\right\}}
$$

where $s(n)$ is the memoryless estimation of $y(n)$ and then the estimation error is:

$$
\varepsilon(n)=y(n) \cdot|y(n)|^{p-2} \cdot\left(R_{p}-|y(n)|^{p}\right)
$$

If we assume that $p=1$, the cost function has the form:

$$
\begin{gathered}
J(n)=E\left\{\left(|y(n)|-R_{1}\right)^{2}\right\} \\
R_{1}=\frac{E\left\{|s(n)|^{2}\right\}}{E\{|s(n)|\}}
\end{gathered}
$$

By rewriting the error signal in (11), (14) can be de- rived.

$$
\varepsilon(n)=y(n)-\frac{y(n)}{|y(n)|}
$$

Updating equation of weights is:

$$
w(n+1)=w(n)+\mu \cdot\left(1-\frac{1}{|y(n)|}\right) \cdot y^{*}(n) \cdot x(n)
$$

It has been shown that the fastest convergence is obtained by using $p=1$.

\section{New On-Line/Off-Line Proposed Method}

In our proposed algorithm, according to the direction and the velocity of the main signal source, new location of user will be estimated. Also the weights of array will be determined using LMS or CM before arriving at the estimated source location. When source reaches to the new point, the antenna array uses these weights and regulates the radiation pattern. For avoiding the error propagation, it is essential that the exact location of the source be determined and array weights be updated at the next step. Details of the steps of new proposed algorithm are illustrated below:

1) Applying the weighting algorithm (LMS or CM) for first two points and determining the direction of arrival in two points $\theta_{1}$ and $\theta_{2}$, and also obtaining the proper weights. For example, in an 8-element uniform linear array (ULA), $w_{11}, w_{12}, w_{13}, w_{14}, w_{15}, w_{16}, w_{17}, w_{18}$ and $w_{21}, w_{22}, w_{23}, w_{24}, w_{25}, w_{26}, w_{27}, w_{28}$.

2) Predicting the next location of the source ( $\theta$ or $\left.\theta_{e q}\right)$ by using the previous locations of the source. The relative velocity has some effects on the value of the angular locations 1 and 2 . In this paper the direction of the source motion has considered in the simulations. Figure 3 shows two cases. If the source moves parallel to main axis of array, according to (16) the new angular location can be found.

$$
\frac{\theta_{2}-\theta_{1}}{v}=\frac{\theta-\theta_{2}}{v} \Rightarrow \theta=2 \theta_{2}-\theta_{1}
$$

3) Finding the proper weights $w_{1}, w_{2}, w_{3}, w_{4}, w_{5}, w_{6}, w_{7}, w_{8}$ for adjusting the radiation pattern in the direction of $\theta$ or $\theta_{e q}$ according to weighting algorithm (LMS or CM).

4) Forming the radiation pattern according to the estimated weights in step 3 .

5) Finding the exact angular location ( $\theta$ or $\theta_{e q}$ ) by using LMS or CM algorithm in the time interval between two sampling points (to avoid error propagation). Then determining the new relative velocity of the source.

6) Going to step 2 and doing the steps 2 to 5 . 


\begin{tabular}{lll}
$\theta_{1}$ & $\theta_{2}$ & $\theta$ \\
\hline
\end{tabular}

(a)

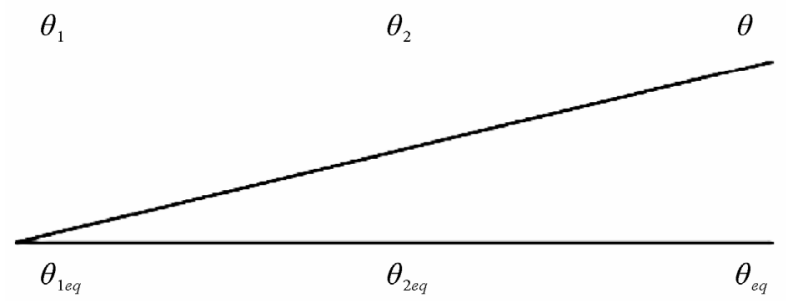

(b)

Figure 3. Movement path of the source respect to main axis of the array. (a) Parallel; b) With nonzero angle.

These 6 steps are summarized as a simulation flowchart in Figure 4.

It can be seen that except first and second points, the other steps of new LMS-based and CM-based algorithms are done in the time interval between two sampling points. Therefore in each beamforming step, radiation pattern just will be activated and other processes are off-line. Table 1 shows the off-line and online processes of conventional and new proposed on-line/off-line beamformers.

\section{Simulations}

In this section, first numerical results of LMS algorithm at different SNRs for three cases, noisy channel, channel with one interferer, and channel with two interferers in both conventional and new proposed beamformers are shown. Second, numerical results belong to simulation of $\mathrm{CM}$ algorithm at different SNRs for three above mentioned channels in both conventional and new proposed beamforming algorithms are illustrated. Here, the accuracy of source angle detection is the performance metric and different simulations are done by changing the angle of source signal in the interval of $\left[-80^{\circ}, 80^{\circ}\right]$.

All simulations are run in MATLAB 7.5 package. Required number of iterations to access acceptable results is 1000 . Three scenarios, pure noisy, one interference signal with angle of zero, and two interference signals in 0 and -40 degrees in 8-element uniform linear antenna array are simulated.

Signal source moves in a defined path with constant velocity. Three paths are considered, parallel to array, in the direction of nonzero angle with array, and random walk with constant velocity. In all simulations, average relative angular velocity is less than 5 degrees in second and signals are modulated using minimum shift keying (MSK) method.

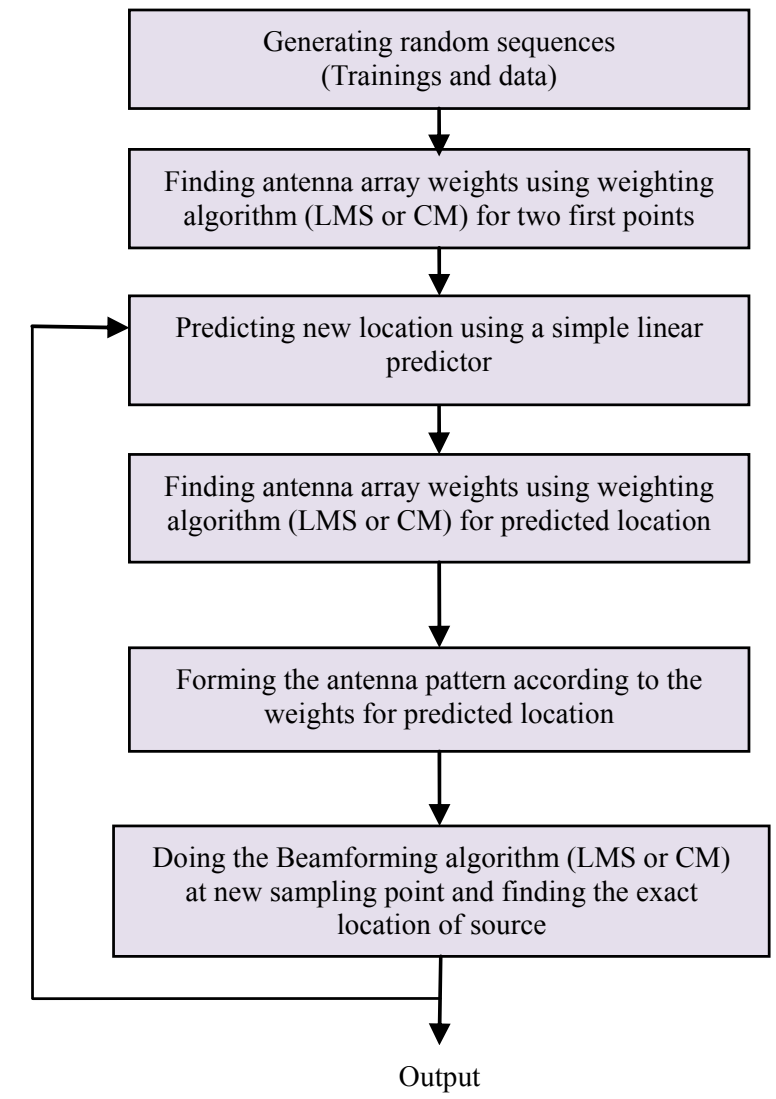

Figure 4. Simulation flowchart of proposed on-line/off-line beamformer.

Table 1. Required off-line and on-line processes for both conventional and new proposed beamformers.

\begin{tabular}{cll}
\hline Process & \multicolumn{1}{c}{$\begin{array}{c}\text { Proposed } \\
\text { Beamformer }\end{array}$} & $\begin{array}{c}\text { Conventional } \\
\text { Beamformer }\end{array}$ \\
\hline \multirow{2}{*}{ Off-line } & $\begin{array}{l}\text { Estimating the velocity } \\
\text { - Updating the predicted loca- } \\
\text { tion of source } \\
\text { - Finding the weights associ- } \\
\text { ated to the next location us- } \\
\text { ing LMS or CM }\end{array}$ & \\
On-line & $\begin{array}{l}\text { Antenna beamforming accor- } \\
\text { ding to calculated weights in } \\
\text { off-line phase }\end{array}$ & $\begin{array}{l}\text { LMS or CM. } \\
\text { Applying the weights to } \\
\text { form the antenna pat- } \\
\text { tern }\end{array}$ \\
\hline
\end{tabular}

In Figure 5 the angular error of both conventional and proposed LMS-based beamformers at different SNRs in the case of noisy channel is shown. As depicted in this figure, the angular accuracy of proposed algorithm is very similar to conventional algorithm. Also Figures 6 and 7 show the angular error of two above mentioned beamformers in terms of SNR in the 1-interference and 2 - interference channels, respectively.

Figures 8, 9 and 10 show the angular error of both con- 


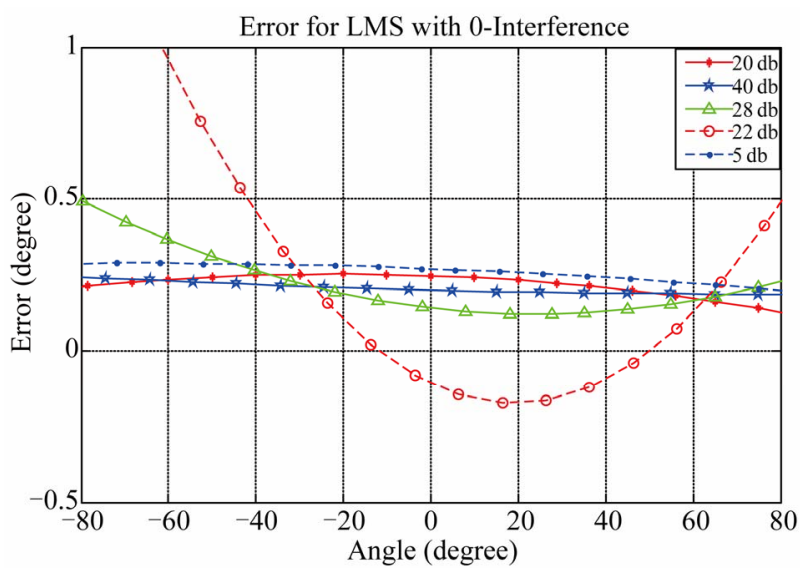

(a)

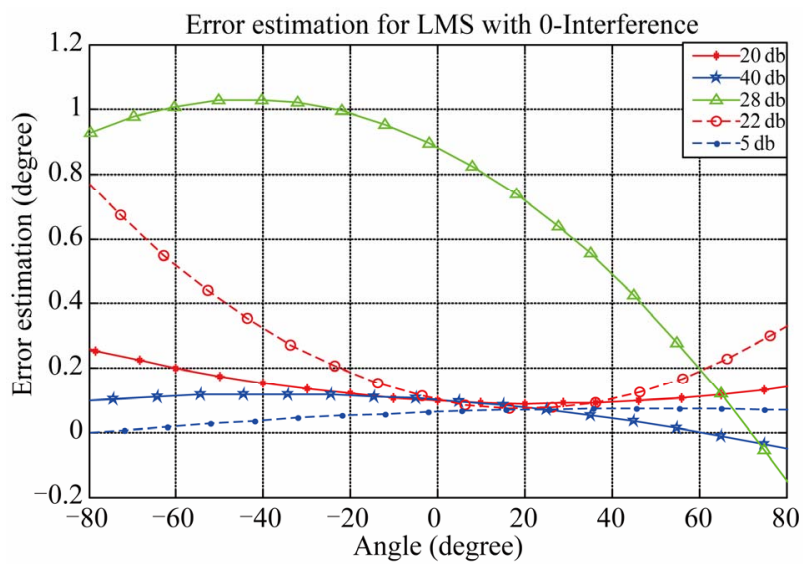

(b)

Figure 5. Angular error of LMS-based beamformers in a noisy channel. (a) Conventional; (b) Proposed on-line/off-line.

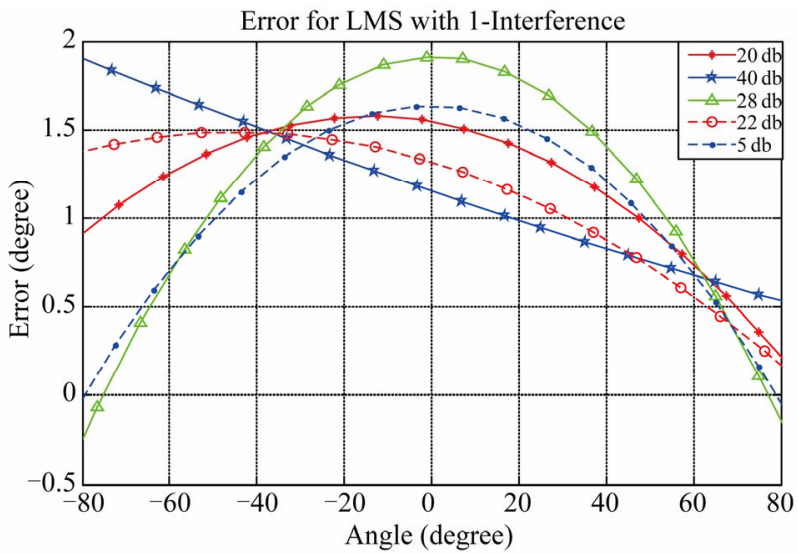

(a)

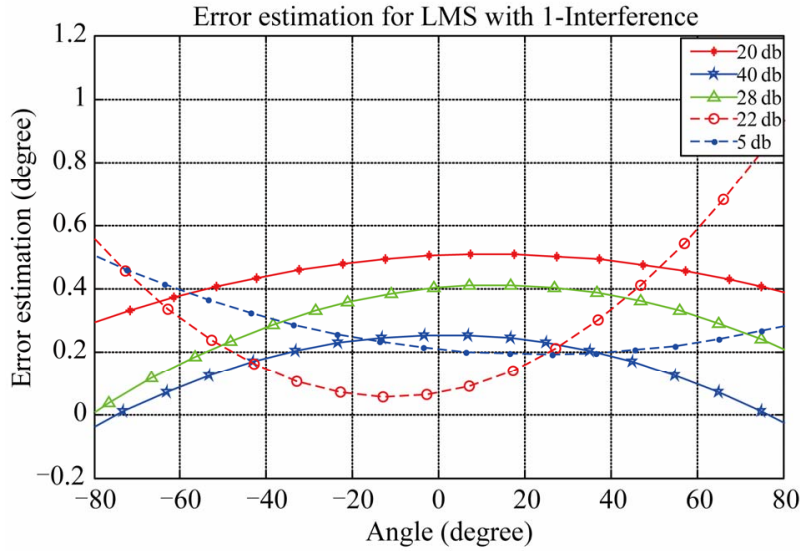

(b)

Figure 6. Angular error of LMS-based beamformers in the case of one interferer. (a) Conventional; (b) Proposed on-line/ off-line.

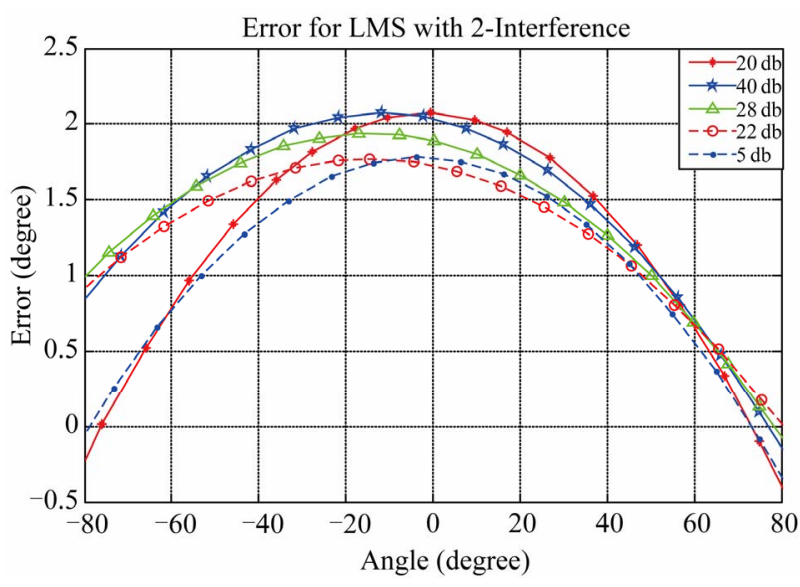

(a)

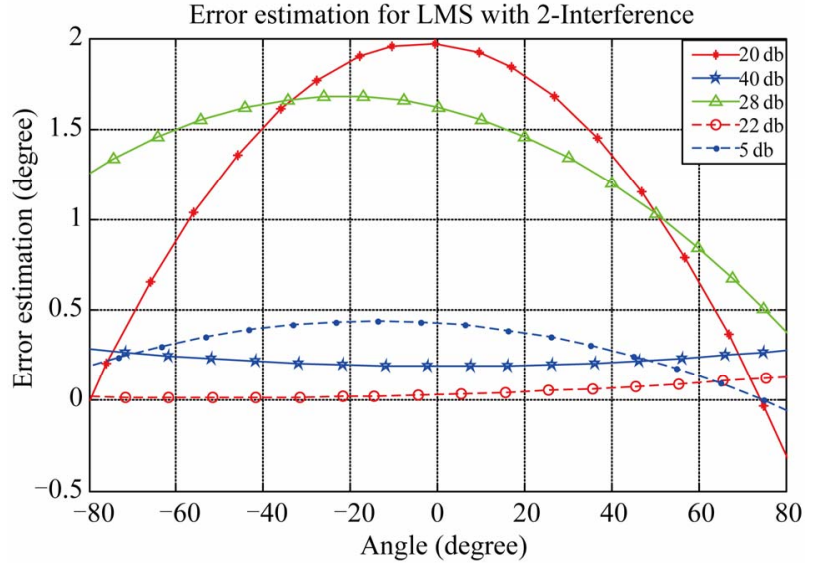

(b)

Figure 7. Angular error of LMS-based beamformers in the case of two interferers. (a) Conventional; (b) Proposed on-line/ off-line. 


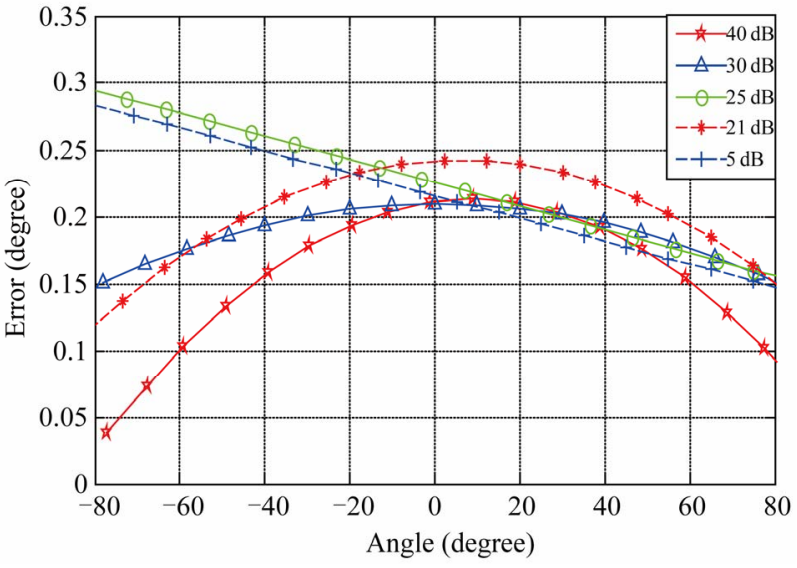

(a)

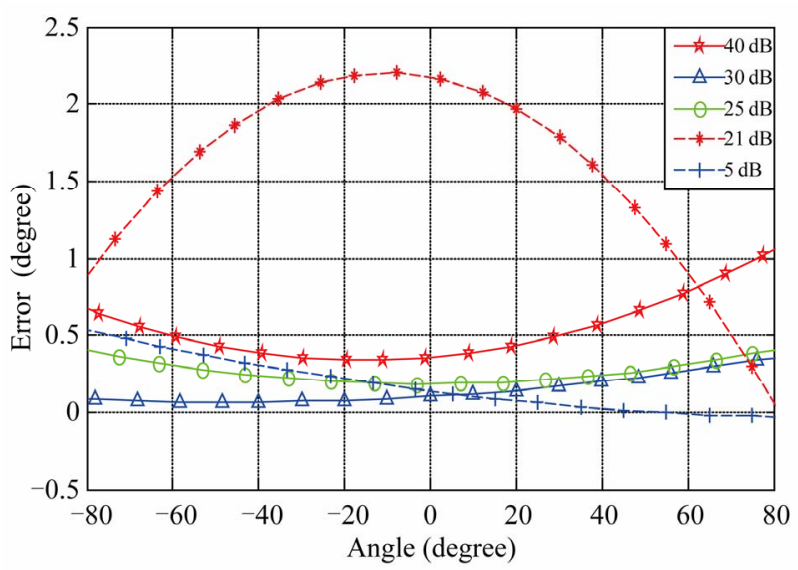

(b)

Figure 8. Angular error of CM-based beamformers in a noisy channel. (a) Conventional; (b) Proposed on-line/off-line.

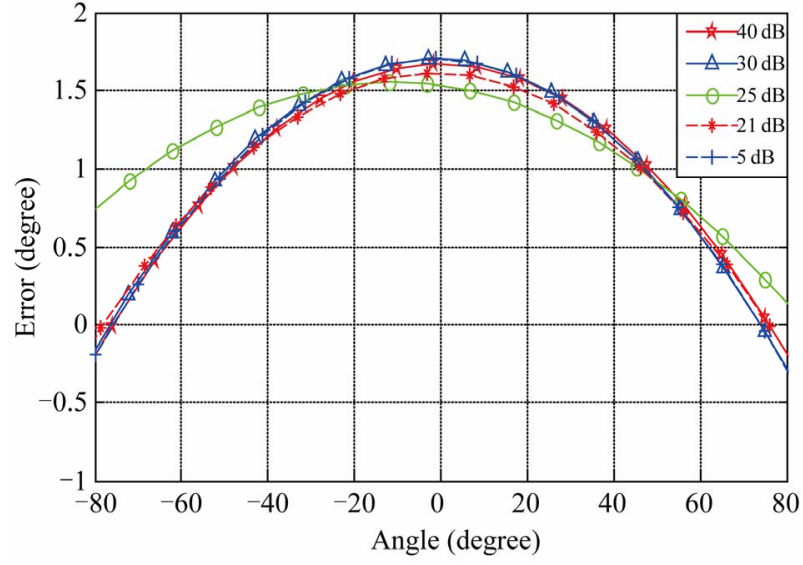

(a)

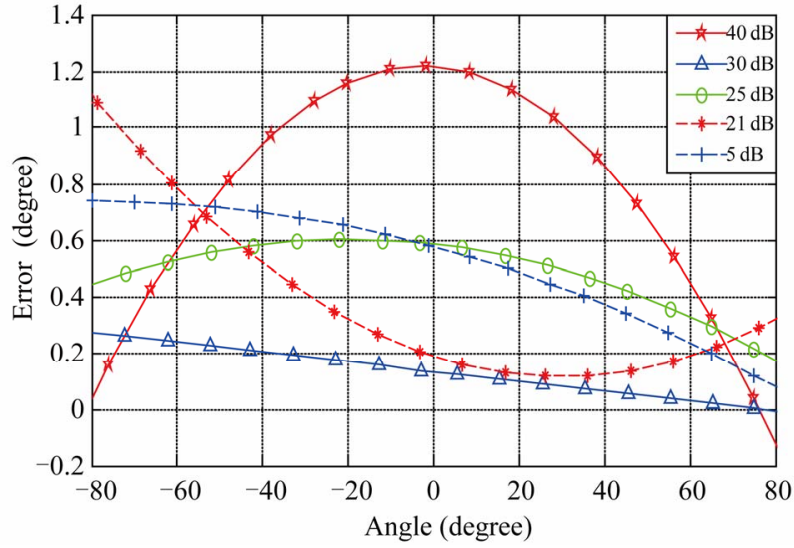

(b)

Figure 9. Angular error of CM-based beamformers in the case of one interferer. (a) Conventional; (b) Proposed on-line/ off-line.

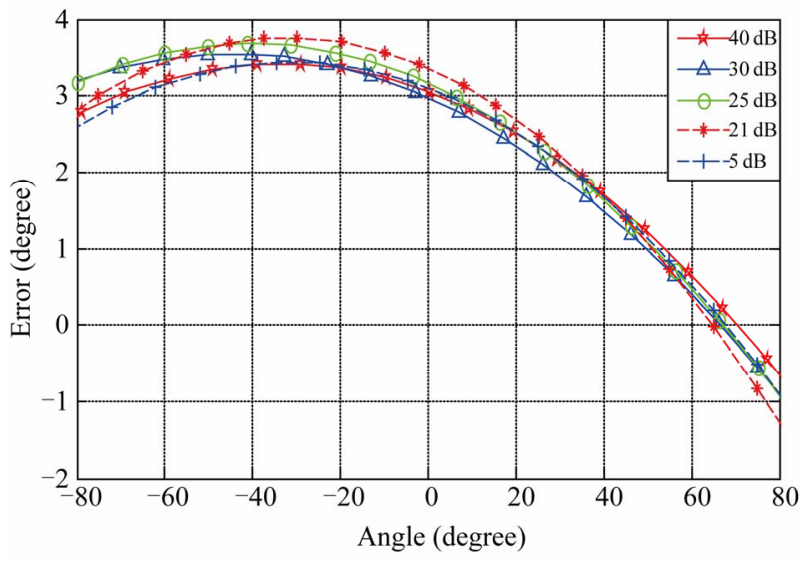

(a)

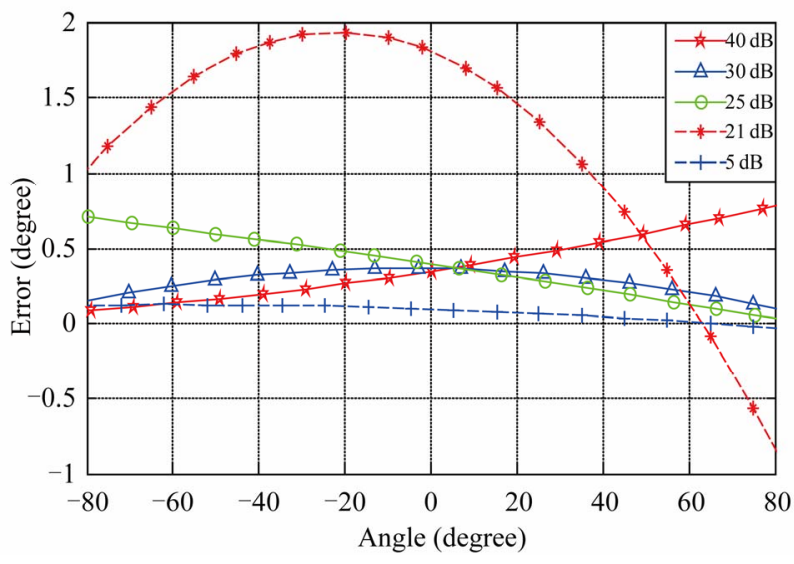

(b)

Figure 10. Angular error of CM-based beamformers in the case of two interferers. (a) Conventional; (b) Proposed on-line/ off-line. 
ventional and proposed CM-based beamformers at different SNRs in three cases, noisy channel, channel with one interferer and channel with two interferers, respectively. As depicted in these figures, the angular accuracy of proposed algorithm is better than conventional one, especially in channels with one and two interferers.

It should be noted that these simulations can be extended to higher number of array elements and array configurations. Besides above mentioned simulation results, it is obvious that in the new proposed beamformer all of processes are separated in two phases, one in the time interval between two points and second in the time of pattern shaping. It means there is no additional calculation for determining the array weights and hence new beamformer offers lower complexity in beamforming time.

\section{Conclusions}

In this paper a novel LMS-based/CM-based beamformer for estimating the weights of adaptive antenna array is proposed. According to this new beamformer, the direction and relative velocity of the source will be estimated based on two previous points and hence the new location of the source will be predicted. According to predicted location, the new weights will be found. These weights are used in the new sampling time. By calculating the accurate weights in the time interval of two consecutive times and updating them, the error propagation effects can be avoided.

In the proposed LMS-based and CM-based algorithms the on-line computational complexity is very lower than associated conventional LMS-based and CM-based algorithms. The inaccuracy in estimated angle of source is less than 1 degree at pure noisy channel and less than 2 degrees in the channel with one and two interfering signals. This algorithm can be used for tracking in radar systems and sensor networks and also regulating the radiation pattern in mobile applications.

\section{Acknowledgements}

This work has been supported by Shahid Rajaee Teacher Training University (SRTTU) under contract number 316 (16.1.1390). We would like to thank anonymous reviewers for their careful reviews of the article. Their comments have certainly improved the quality of this article. Also, the authors would like thank Mrs. Samaneh Movassaghi, Sydney University of Technology, for the selfless help she provided.

\section{References}

[1] S. Shirvani-Moghaddam and M. Shirvani-Moghaddam,
"A Comprehensive Survey on Antenna Array Signal Processing," Journal of Trends in Applied Science Research, Vol. 6, No. 6, 2011, pp. 507-536. doi:10.3923/tasr.2011.507.536

[2] C. A. Balanis and P. I. Ioannides, "Introduction to Smart Antennas," Morgan \& Claypool, San Rafael, 2007.

[3] T. K. Sarkar, M. C. Wicks, M. Salazar-Palma and R. J. Bonneau, "Smart Antennas," John Wiley and Sons, Hoboken, 2003.

[4] C. Sun, J. Cheng and T. Ohira, "Handbook on Advancements in Smart Antenna Technologies for Wireless Networks," Idea Group Inc., Hershey, 2009.

[5] J. Fuhl and E. Bonek, "Temporal Reference Algorithms versus Spatial Reference Algorithms for Smart Antennas," Wireless Personal Communications, Vol. 9, No. 3, 1998, pp. 271-293. doi:10.1023/A:1018332029467

[6] S. Haykin, "Adaptive Filter Theory," Prentice Hall, Upper Saddle River, 1996.

[7] A. Bouacha, F. Debbat and F. T. Bendimerad, "Modified Blind Beamforming Algorithm for Smart Antenna System," Journal of Radio Electronics, No. 1, 2008.

[8] P. C. Parini, D. X. Chen, D. J. Bigham, P. I. Liewellyn, D. L. Samuel, D. L. Ho and B. Collins, "Final Report on Semi-Smart Antenna Technology Project," 2nd Edition, BSC Associates Ltd., Semi-Smart Antenna Project, Ofcom Project No. 830000081, July 2006.

[9] J. Homer, P. J. Kootsookos and V. Selvaraju, "Enhanced NLMS Adaptive Array via DOA Detection," IET Communications Magazine, Vol. 1, No. 1, 2007, pp. 19-26.

[10] X. Sun, X. Lian and J. Zhou, "Robust Adaptive Beamforming Based on Maximum Likelihood Estimation," International Conference on Microwave and Millimeter Wave Technology, Nanjing, 21-24 April 2008, pp. 11371140.

[11] H. Chen, D. Kasilingam, "Performance Analysis of Super-Resolution Bamforming in Smart Antennas," IEEE International Conference on Acoustics, Speech, and Signal Processing, Montreal, 17-21 May 2004, pp. 353-356. doi:10.1109/ICASSP.2004.1327120

[12] R. M. Shubair, M. A. Al-Qutayri and J. M. Sa, "A Setup for the Evaluation of MUSIC and LMS Algorithms for a Smart Antenna System," Journal of Communications, Vol. 2, No. 4, June 2007, pp. 71-77.

[13] C. S. Rani, P. V. Subbaiah, K. C. Reddy and S. S. Rani, "LMS and RLS Algorithms for Smart Antennas in a W-CDMA Mobile Communication Environment," ARPN Journal of Engineering and Applied Sciences, Vol. 4, No. 6, August 2009, pp. 77-88.

[14] M. Islam and Z. Rashid, "MI-NLMS Adaptive Beamforming Algorithm for Smart Antenna System Applications," Journal of Zhejiang University Science A, Vol. 7, No. 10, 2006, pp. 1709-1716. doi:10.1631/jzus.2006.A1709

[15] M. T. Islam and N. Misran, "MI-NLMS Adaptive Beamforming Algorithm with Tracking Ability," Journal of Applied Sciences, Vol. 9, No. 12, 2009, pp. 2335-2339. doi:10.3923/jas.2009.2335.2339 
[16] S. F. Shaukat, M. Hassan, R. Farooq, H. U. Saeed and Z. Saleem, "Sequential Studies of Beamforming Algorithms for Smart Antenna Systems," World Applied Sciences Journal, Vol. 6, No. 6, 2009, pp. 754-758. doi:10.1109/ISWCS.2007.4392422

[17] X. Wang and G. Z. Feng, "Performance Analysis of RLS Linearly Constrained Constant Modulus Algorithm for Multiuser Detection," Elsevier Signal Processing, Vol. 89, No. 2, 2009, pp. 181-186.

[18] L. Wang, R. C. de Lamare and Y. L. Cai, "Low-Complexity Adaptive Step Size Constrained Constant Modulus SG Algorithms for Adaptive Beamforming," El- sevier Signal Processing, Vol. 89, No. 12, 2009, pp. 2503- 2513.

[19] L. Wang and R. C. Lamare, "Constrained Constant Modulus RLS-based Blind Adaptive Beamforming Algorithm for Smart Antennas," 4th International Symposium on Wireless Communication Systems, Trondheim, 17-19 October 2007, pp. 657-661.

[20] H. Sadeghi, S. Shirvani-Moghaddam and V. T. Vakili, "Appropriate CCM-Based Algorithm for Adaptive Antenna Array Beamforming," Proceedings of 5th International Symposium on Telecommunications, Tehran, 4-6 December 2010, pp. 69-75. 